
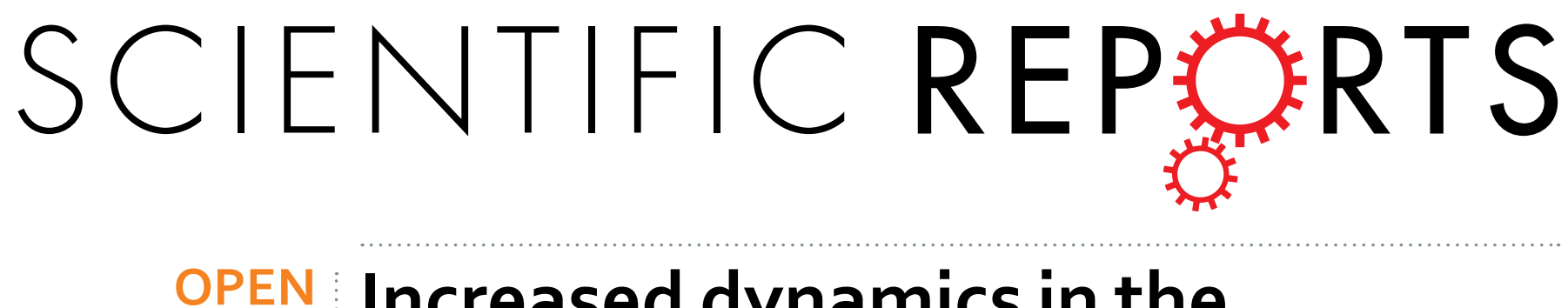

\title{
Increased dynamics in the
}

\section{0-57 $\Omega$-loop of the G41S variant of human cytochrome $c$ promote its pro-apoptotic conformation}

Accepted: 05 July 2016

Published: 27 July 2016

\section{Andreas loannis Karsisiotis ${ }^{1}$, Oliver M. Deacon ${ }^{1}$, Michael T. Wilson ${ }^{1}$, Colin Macdonald ${ }^{2}$, Tharin M. A. Blumenschein ${ }^{2}$, Geoffrey R. Moore ${ }^{2}$ \& Jonathan A. R. Worrall ${ }^{1}$}

Thrombocytopenia 4 is an inherited autosomal dominant thrombocytopenia, which occurs due to mutations in the human gene for cytochrome $c$ that results in enhanced mitochondrial apoptotic activity. The Gly41Ser mutation was the first to be reported. Here we report stopped-flow kinetic studies of azide binding to human ferricytochrome $c$ and its Gly41Ser variant, together with backbone amide $\mathrm{H} / \mathrm{D}$ exchange and ${ }^{15} \mathrm{~N}$-relaxation dynamics using NMR spectroscopy, to show that alternative conformations are kinetically and thermodynamically more readily accessible for the Gly41Ser variant than for the wild-type protein. Our work reveals a direct conformational link between the 40-57 $\Omega$-loop in which residue 41 resides and the dynamical properties of the axial ligand to the heme iron, Met80, such that the replacement of glycine by serine promotes the dissociation of the Met80 ligand, thereby increasing the population of a peroxidase active state, which is a key non-native conformational state in apoptosis.

Mitochondrial cytochrome $c$ (cyt) is a multifunctional protein that can act as an electron carrier in oxidative phosphorylation as a peroxidase in the early stages of the intrinsic apoptosis pathway, as a component of the apoptosome, a key complex in the apoptotic pathway and as a signalling molecule affecting the activity of the nuclear oncoprotein SET/template-activating factor-I $\beta$ (SET/TAF-I $\beta$ ) complex ${ }^{1-4}$. Cyt possesses a hexacoordinate heme iron, where His18 and Met80 serve as the axial heme iron ligands, with the heme encapsulated in a hydrophobic environment created by a polypeptide fold of five $\alpha$-helices and $3 \Omega$-loops (Fig. 1A). Under native conditions ferricyt possesses a low level of peroxidase activity ${ }^{5}$ due to the existence of an equilibrium between the dominant hexacoordinate native form, and a minor populated pentacoordinate form in which Met 80 is not coordinated to the heme iron ${ }^{6}$. Preceding its release from the mitochondrion at the onset of apoptosis, cyt forms a complex with the phospholipid cardiolipin (CL), which drives the formation of the pentacoordinate form and greatly boosts peroxidase activity ${ }^{2,7}$. Further to this, at alkaline $\mathrm{pH}$, ferricyt can access a conformational state whereby a Lys residue ( 73 or 79 ) within the $71-85 \Omega$-loop replaces the native Met 80 ligand ${ }^{8}$. This alkaline isomerisation occurs with an apparent $\mathrm{pK}$ of $\sim 8-9$, depending on solution conditions and temperature ${ }^{9}$. Thus ferricyt has several energetically accessible conformational states with very different properties from the native state.

Thrombocytopenia 4 (THC4; OMIM 612004) is an inherited autosomal disease, which occurs due to mutations in the human cytochrome $c$ gene (CYCS) resulting in enhanced mitochondrial apoptotic activity ${ }^{10}$. Two mutations in the CYCS gene have been identified that produce the G41S and Y48H variants of human cytochrome $c(\mathrm{H} \text {-cyt })^{10,11}$. The G41S and $\mathrm{Y} 48 \mathrm{H}$ substitutions are located in the $40-57 \Omega$-loop ${ }^{12-15}$, which has the lowest free energy of the five cooperative folding/unfolding units (foldons) assigned in cyt ${ }^{16,17}$ (Fig. 1A). In the absence of CL the G41S variant has been shown to have increased peroxidase activity relative to the wild-type (WT) protein $^{18,19}$. High-resolution X-ray structures for the WT and G41S variant of H-cyt have been determined ${ }^{20,21}$ with no gross structural differences between them detected. However, subtle differences in a hydrogen-bond network in the vicinity of the heme propionate- 7 substituent were observed ${ }^{21}$ (Fig. 1B) and these may be significant as the electronic properties of the heme propionates are known to affect heme reactivity ${ }^{22}$. The increase in polarity 
A

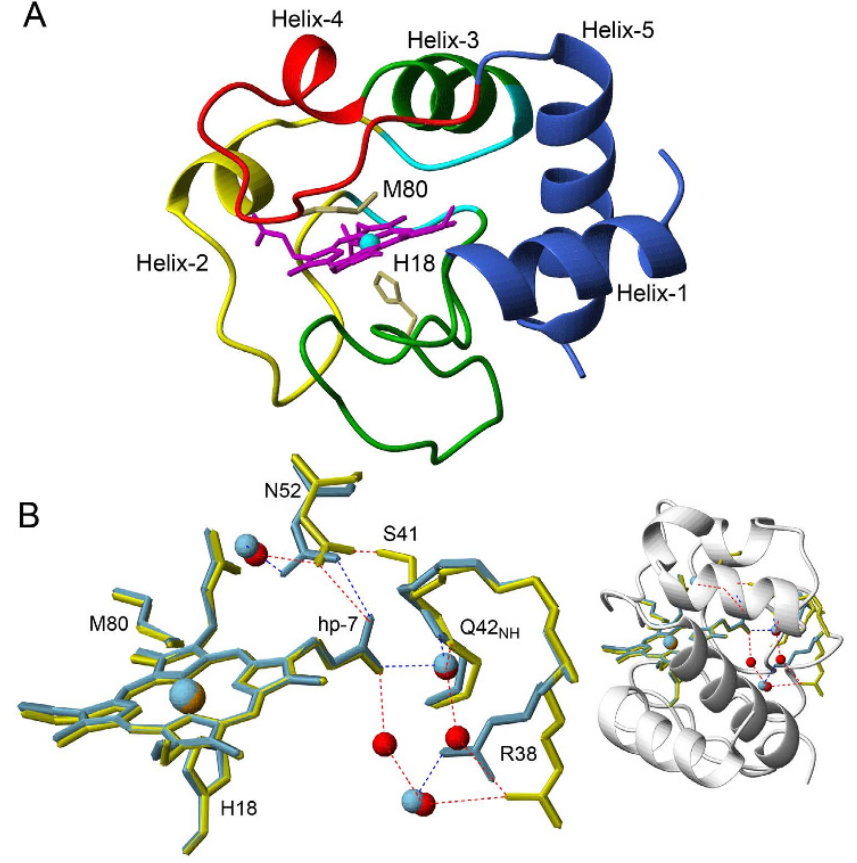

Figure 1. Structure of human cytochrome $c$. (A) Cartoon representation of $\mathrm{H}$-cyt with the five foldons identified in horse cyt indicated and coloured as follows: the blue unit ( $\mathrm{N}$ and $\mathrm{C}$-terminal helices, residues 1-13, 86-104), the green unit (residues 14-36 $\Omega$-loop, 62-70), the cyan neck unit (residues 37-39 and 58-61), the red unit (residues 71-85 $\Omega$-loop) and the yellow unit (residues 40-57 $\Omega$-loop). The His 18 and Met80 ligands are labelled (PDB code: $3 Z C F)^{21}$. (B) Positional movement of the Arg38 and Asn52 side chains identified in the G41S variant (yellow, PDB code: $3 \mathrm{NWV})^{20}$ compared to the WT protein (light blue, PDB code: $\left.3 \mathrm{ZCF}\right)^{21}$. $\mathrm{H}$-bond networks are illustrated as blue (WT) and red broken lines (G41S), with $\mathrm{H}_{2} \mathrm{O}$ molecules shown as light blue (WT) and red (G41S) spheres. Note that in the G41S structure two additional $\mathrm{H}_{2} \mathrm{O}$ molecules neighbour Arg38.

of the heme environment of the G41S protein resulting from the movement of Arg38 and the incorporation of additional water molecules (Fig. 1B) may also contribute to an ease of access of $\mathrm{H}_{2} \mathrm{O}_{2}$ to the heme of the G41S variant leading directly to its enhanced peroxidase activity ${ }^{19,23}$. In addition to the small conformational difference between WT and the G41S variant there is a substantial difference in global stability with the free energy of denaturation of WT H-ferricyt at $10.5 \mathrm{kcal} \mathrm{mol}^{-1}$ being $2.6 \mathrm{kcal} \mathrm{mol}^{-1}$ greater than that of the G41S variant ${ }^{21}$.

Based on the inherent ability of ferricyt to access non-native conformations, it is a possibility that the G41S variant perturbs the dynamics of the $\mathrm{H}$-ferricyt energy landscape, thereby changing the relative populations and kinetics of conversion between the native state and a non-native conformer. Solution state NMR spectroscopy is well suited to provide insight at atomic resolution on the dynamics and conformational changes that serve to regulate function in biomolecules ${ }^{24,25}$. Past NMR investigations have probed the backbone dynamics of cyt with the rationale of assessing whether differences in dynamics exist between the heme redox states ${ }^{26-28}$. In addition to NMR the dynamics of cyt are open to investigation by a variety of heme-centred approaches, particularly with ligand binding studies ${ }^{6,29}$. From such studies, combined with NMR data, a consensus exists that the energy landscapes of mitochondrial cyt differ considerably between redox states with the ferric form being more flexible ${ }^{22,27}$ and having a lower stability than the ferrous form ${ }^{22,30}$. This may have functional implications for cyt function in apoptosis, as it is the ferric oxidation state that has been demonstrated to be necessary for interaction with key components of the apoptotic pathway such as the Apoptotic protease activating factor 1 (Apaf-1) $)^{31}$ and also to facilitate peroxidase activity ${ }^{32}$.

In the present study we have used azide $\left(\mathrm{N}_{3}{ }^{-}\right)$binding to the heme iron of $\mathrm{H}$-ferricyt, backbone amide proton $\mathrm{H} / \mathrm{D}$ exchange and ${ }^{15} \mathrm{~N}$ relaxation dynamics of the oxidised protein to assess whether the G41S variant has altered dynamics compared to WT H-ferricyt. We find that the population of the pentacoordinate form is significantly greater for the G41S variant than the WT protein, and that the G41S variant has distinctly enhanced dynamics, particularly in the 40-57 $\Omega$-loop foldon, which give rise to the increased lability of the Met 80 ligand. These findings offer a direct structural insight into how main chain dynamics relate to key structural properties of the heme iron of $\mathrm{H}$-ferricyt and importantly how the G41S variant is proapoptotic ${ }^{10,19,21,23}$ in the context of thrombocytopenia 4.

\section{Results and Discussion}

The pentacoordinate heme form is more populated in the G41S variant. Exogenous ligand binding to ferricyt can be used as a diagnostic probe to assess the lability of the Met80 ligand. On mixing $\mathrm{N}_{3}{ }^{-}$with WT or G41S H-ferricyt an optical transition was observed (Fig. 2A) with differing time courses for the two proteins, 

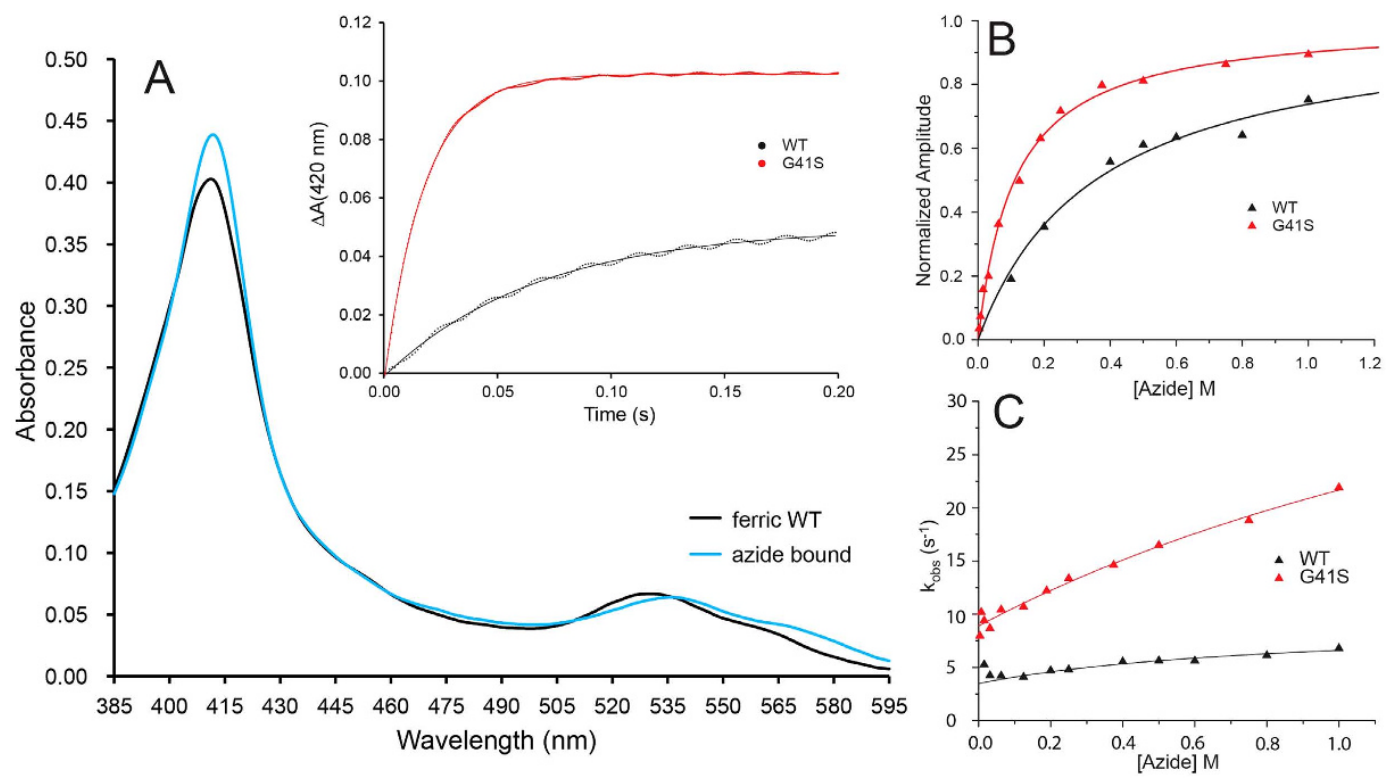

Figure 2. Stopped-flow kinetics of $\mathrm{N}_{3}{ }^{-}$binding to $\mathrm{H}$-ferricyt at $\mathrm{pH} 7$ and $25^{\circ} \mathrm{C}$. (A) Spectra generated on mixing $\mathrm{H}$-ferricyt with $\mathrm{N}_{3}^{-}$. Global analysis indicated a single transition with the initial spectrum (black) and the $\mathrm{N}_{3}{ }^{-}$bound spectrum (light blue). Inset: time courses for $\mathrm{N}_{3}{ }^{-}$binding to WT (black) and G41S (red) $(5 \mu \mathrm{M}$ after mixing) are shown together with exponential fits. (B) The normalised amplitudes of the time courses recorded at $420 \mathrm{~nm}$ plotted against $\left[\mathrm{N}_{3}^{-}\right]$. Binding curves are fitted to a hyperbolic equation to yield the $K_{\text {app }}$ values reported in the main text. (C) The rate constant $\left(\mathrm{k}_{\mathrm{obs}}\right)$ determined for $\mathrm{N}_{3}{ }^{-}$binding with solid lines representative of fits to Eq. 2, to give the $\mathrm{k}_{1}$ and $\mathrm{k}_{-2}$ values reported in the main text.

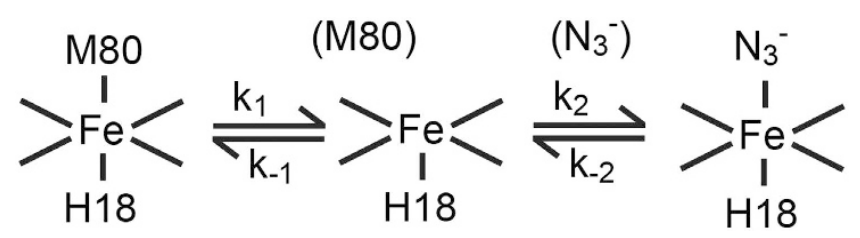

Figure 3. SN1 mechanism of $\mathrm{N}_{3}{ }^{-}$binding to $\mathrm{H}$-ferricyt. The dissociation and re-association of the Met80 ligand to the $\mathrm{Fe}(\mathrm{III})$ occur with rates of $\mathrm{k}_{1}$ and $\mathrm{k}_{-1}$, respectively, prior to $\mathrm{N}_{3}{ }^{-}$association.

as shown in the inset to Fig. 2A. Such an optical transition reflects the dissociation of the Met80 ligand from the heme and the binding of $\mathrm{N}_{3}{ }^{-}$in its place. Confirmation of this assignment was obtained in separate experiments at higher [H-ferricyt] (Fig. S1) that revealed the addition of $\mathrm{N}_{3}^{-}$to WT and G41S led to the bleaching of the $695 \mathrm{~nm}$ band, which is diagnostic for the loss of the Met80 ligand. The optical transition consisted of a single exponential at every wavelength with no intermediates detected (inset Fig. 2A). The normalised amplitudes of such time courses, taken over a range of $\left[\mathrm{N}_{3}{ }^{-}\right]$conform to a simple hyperbolic binding isotherm (Fig. 2B) to yield apparent equilibrium dissociation constants $\left(K_{\mathrm{app}}\right)$ of $0.31 \pm 0.03$ and $0.11 \pm 0.01 \mathrm{M}$ for WT and G41S H-ferricyt, respectively. The dependence of the observed rate constant $\left(\mathrm{k}_{\mathrm{obs}}\right)$ for $\mathrm{N}_{3}{ }^{-}$binding obtained from plots such as those in the inset to Fig. 2A is under the $\left[\mathrm{N}_{3}{ }^{-}\right.$] measureable, almost linear (vide infra) for both WT and G41S (Fig. 2C). Since the apparent $\mathrm{p} K$ values for the alkaline transitions of WT and G41S H-ferricyt are different, at 9.3 and 8.2, respectively, $\mathrm{N}_{3}{ }^{-}$binding experiments at higher $\mathrm{pH}$ values (see Figs S2 and S3) were conducted and revealed that the alkaline transition is not significant for the binding of $\mathrm{N}_{3}{ }^{-}$to $\mathrm{H}$-ferricyt at $\mathrm{pH}$ 7. It is therefore concluded that $\mathrm{N}_{3}{ }^{-}$binding to the G41S variant occurs faster and with a higher binding affinity than it does to WT H-ferricyt.

Ligand binding to the heme iron of ferricyt is generally discussed in terms of an SN1 mechanism as depicted in Fig. 3, where the hexacoordinate ferricyt is in equilibrium with a pentacoordinate form in which the Met80 is dissociated from the heme. This equilibrium lies strongly in favour of the hexacoordinate form, with $\mathrm{N}_{3}{ }^{-}$binding to the pentacoordinate form yielding the low-spin hexacoordinate $\mathrm{N}_{3}{ }^{-}$adduct (Fig. 3). The mechanism given is amenable to full analysis but this leads to unwieldy equations to describe the kinetic behaviour of the system. However, a simplifying assumption can be made in that the concentration of the pentacoordinate species, that is at all times small, is in a steady-state throughout the reaction ${ }^{29}$. Furthermore, as the $\left[\mathrm{N}_{3}{ }^{-}\right]$is high relative to the [H-ferricyt] the binding kinetics may be considered to be pseudo-first order. These simplifications allow for equations 1 and 2 to be derived that describe the $K_{\text {app }}$ (Eq. 1) and the dependence of $\mathrm{k}_{\mathrm{obs}}$ for $\mathrm{N}_{3}{ }^{-}$binding as a function of $\left[\mathrm{N}_{3}^{-}\right]$(Eq. 2). 


$$
\begin{gathered}
K_{a p p}=K_{D}(K+1 / K) \\
k_{o b s}=\frac{\left(k_{1}-k_{-2}\right)\left[N_{3}^{-}\right]}{\left(k_{1}+k_{-1} / k^{\prime}\right)+\left[N_{3}^{-}\right]}+k_{-2}
\end{gathered}
$$

where $\mathrm{K}=\mathrm{k}_{1} / \mathrm{k}_{-1}$ and $\mathrm{K}_{\mathrm{D}}=\mathrm{k}_{-2} / \mathrm{k}_{2}$, with $\mathrm{k}_{2}$ the pseudo-first order rate constant for $\mathrm{N}_{3}{ }^{-}$binding i.e. $\mathrm{k}_{2}=\mathrm{k}^{\prime}\left[\mathrm{N}_{3}{ }^{-}\right], \mathrm{k}^{\prime}$ the second order rate constant and $\mathrm{k}_{\mathrm{obs}}$ is $\sim \mathrm{k}_{1}$ at high $\left[\mathrm{N}_{3}^{-}\right.$]. Equation 1 shows that if $\mathrm{K}<1$ (as here where the pentacoordinate concentration is very low) then $K_{\text {app }}$ is $\sim \mathrm{K}_{\mathrm{D}} / \mathrm{K}$. Assuming that $\mathrm{N}_{3}{ }^{-}$binding to the pentacoordinate species is not directly affected by a distant mutation in the protein, then $K_{\mathrm{D}}$ will be the same for both proteins and the ratio of $K_{\mathrm{app}}$ values $\left({ }^{\mathrm{WT}} \mathrm{K}_{\mathrm{app}} /{ }^{\mathrm{G} 41 \mathrm{~S}} \mathrm{~K}_{\mathrm{app}}\right.$ ) reflect the ratio of $\mathrm{K}$ values $\left({ }^{\mathrm{G} 41 \mathrm{~S}} \mathrm{~K} /{ }^{\mathrm{WT}} \mathrm{K}\right)$. On this basis ${ }^{\mathrm{G} 41 \mathrm{~S}} \mathrm{~K}>{ }^{\mathrm{WT}} \mathrm{K}$ and thus the pentacoordinate form is more populated in the G41S variant than in the WT protein.

Equation 2 predicts that plots of $\mathrm{k}_{\mathrm{obs}}$ versus $\left[\mathrm{N}_{3}{ }^{-}\right.$] follow hyperbolae that intercept the $\mathrm{k}_{\mathrm{obs}}$ axis at the value of $\mathrm{k}_{-2}$, the $\mathrm{N}_{3}{ }^{-}$dissociation rate constant, and plateau at $\mathrm{k}_{1}$, the Met80 dissociation rate constant. Fitting the data in Fig. $2 \mathrm{C}$ to Eq. 2 gives $\mathrm{k}_{-2}$ values of $3.5 \pm 0.4$ and $8.9 \pm 0.3 \mathrm{~s}^{-1}$ for WT and G41S, respectively. Over the [ $\mathrm{N}_{3}{ }^{-}$] range that was accessible experimentally the hyperbolic curvature was not discerned and thus reliable values of the Met80 dissociation constants $\left(\mathrm{k}_{1}\right)$ cannot be provided. However, it is possible to estimate minimum values for $\mathrm{k}_{1}$ of $5.77 \pm 1.5$ and $45 \pm 18 \mathrm{~s}^{-1}$, for WT and G41S, respectively, consistent both with Eq. 2 and the data (Fig. 2C). The $\mathrm{k}_{1}$ for $\mathrm{H}$-ferricyt may be compared to the value of $11-16 \mathrm{~s}^{-1}$ for horse ferricyt obtained by non-linear fitting procedures of the data reported by Sutin and Yandell using $\mathrm{N}_{3}{ }^{-}$as the competing ligand ${ }^{6}$. The differences between horse and $\mathrm{H}$-ferricyt is possibly associated with the sequence difference at position 83 in the $71-85 \Omega$-loop that may effect the dynamics of the Met80 ligand as proposed by Bowler and co-workers ${ }^{33}$. Thus the considerably larger estimates for the rate of Met80 dissociation for the G41S variant is again consistent with the pentacoordinate form being more populated in the G41S variant than in the WT protein, pointing towards a more flexible and labile heme crevice.

The lowest free energy cyt foldon has increased H/D-exchange in the G41S variant. Monitoring $\mathrm{H} / \mathrm{D}$ exchange for backbone amide protons with NMR spectroscopy is a tool that provides per residue resolution of structural features and thermodynamic stability of a protein, with exchange rates influenced mainly by hydrogen bonding, particularly in secondary structure, and solvent accessibility ${ }^{34}$. A convenient form of expressing variation in $\mathrm{H} / \mathrm{D}$ exchange behaviour is to compare the amide protection actors (PF), defined as the ratio between the observed experimental and intrinsic exchange rates $\left(\mathrm{k}_{\mathrm{ex}} / \mathrm{k}_{\mathrm{in}}\right)$, where the intrinsic exchange rates are the rates of exchange for the given amino acid sequence, $\mathrm{pH}$ and temperature determined using unstructured peptides ${ }^{35}$. Table S1 reports the exchange rates, calculated PFs, free energies of exchange $\left(\Delta G_{e x}\right)$ and proton occupancy values for each residue. For both WT and G41S H-ferricyt, 32 and 46 amides, respectively, are fully exchanged within the first $10 \mathrm{~min}$. Figure 4A shows the sequence dependence of the PFs for the 63 remaining residues of WT for which $\mathrm{H} / \mathrm{D}$ exchange rates could be calculated and Fig. 4B, the comparable figure for the 44 residues for which $\mathrm{H} / \mathrm{D}$ exchange data could be determined for the G41S variant. The H/D exchange profile of WT can be broadly divided into six continuous regions of increased protection, residues 6-15, 32-45, 51-54, 64-70, 73-75 and 90-103 (Fig. 4A). With the exception of segment 32-45, the protected regions correlate well with the presence of $\alpha$-helical secondary structure (Fig. 5A). This profile is largely retained for the G41S variant, however the helix 2 region (residues 51-54) and residues between 27-43 are notable exceptions (Fig. 4B). As is clear from Fig. 5B, many of the residues in the G41S variant having reduced protection to $\mathrm{H} / \mathrm{D}$ exchange are close to the heme on the Met80 side of the heme. This is consistent with the results of the $\mathrm{N}_{3}{ }^{-}$binding experiments, which shows that there is a greater amount of the pentacoordinate form for the G41S variant.

The H/D exchange data for $\mathrm{H}$-ferricyt is comparable to that reported for yeast and horse ferricyt ${ }^{36,37}$. Protons showing the slowest exchange are mostly confined to the $\mathrm{N}$ - and C-terminal foldon ${ }^{37,38}$. In general, $\mathrm{H} / \mathrm{D}$ exchange for amides that are in $\mathrm{H}$-bonds in the ground-state structure of a protein can occur via global unfolding of the protein, through local unfolding, or by a combination of the two. An indicator that global unfolding is the key event is shown by a correspondence between the $\Delta \mathrm{G}_{\text {stability }}$ as determined from denaturant-induced equilibrium unfolding, and $\Delta \mathrm{G}_{\mathrm{ex}}$ for the amide $\mathrm{H} / \mathrm{D}$ exchange process. When $\Delta \mathrm{G}_{\text {stability }}>\Delta \mathrm{G}_{\mathrm{ex}}$ local unfolding promotes the $\mathrm{H} / \mathrm{D}$ exchange. This is the case in horse ferricyt where most of the amides involved in $\mathrm{H}$-bonds in the ground-state structure have $\Delta \mathrm{G}_{\text {stability }}>\Delta \mathrm{G}_{\mathrm{ex}}$ and thus local unfolding is important for this protein ${ }^{36}$. This is also the case here for WT H-ferricyt as $\Delta \mathrm{G}_{\text {stability }}$ measured under the same conditions as the H/D exchange (Table S2) is significantly greater than the $\Delta \mathrm{G}_{\text {ex }}$ values given in Table S1. Maity et al.${ }^{39}$ describe H/D exchange in horse cyt in terms of five foldon units that fold with different folding free energies in a stepwise sequential manner. The foldon with the greatest stability corresponds to the $\mathrm{C}$-terminal and $\mathrm{N}$-terminal $\alpha$-helices, whilst the two foldons with the lowest stability are the 71-85 and 40-57 $\Omega$-loops both involving residues packed around the heme (Fig. 1A $)^{16,39}$. Our $\mathrm{H} / \mathrm{D}$ exchange data for $\mathrm{H}$-ferricyt are broadly in agreement with their foldon model (Figs 4 and 5).

While WT and G41S H-ferricyt share a broadly similar H/D exchange profile, differences are apparent (Fig. 4C). The decreased level of protection in the G41S variant maps onto the three lowest stability foldons (Fig. 1A) ${ }^{39}$ and are assigned to residues 34-43 (the neck), 51-54 (40-57 $\Omega$-loop), 59 and 60 (the neck), 73-75, 79 and 80 (71-85 $\Omega$-loop) (Fig. 5C), which predominately encompass the heme propionate-7 region of the protein, whose conformation is altered in the G41S variant (Fig. 1B) ${ }^{21}$. The most affected residues of G41S in terms of increased susceptibility to H/D exchange are Arg38 and Trp59 (the neck), Ile75 (71-85 $\Omega$-loop) and their near neighbours, but Lys27 and Gly34 (14-36 $\Omega$-loop), Ser41, Asp51, Asn52, Lys53 (40-57 $\Omega$-loop), and Lys79 and Met80 (71-85 $\Omega$-loop) also stand out with a significantly diminished H/D exchange protection compared to the corresponding residues of WT (Fig. 4C). Residues Asn52 and Trp59 are conserved in 99\% of the mitochondrial 

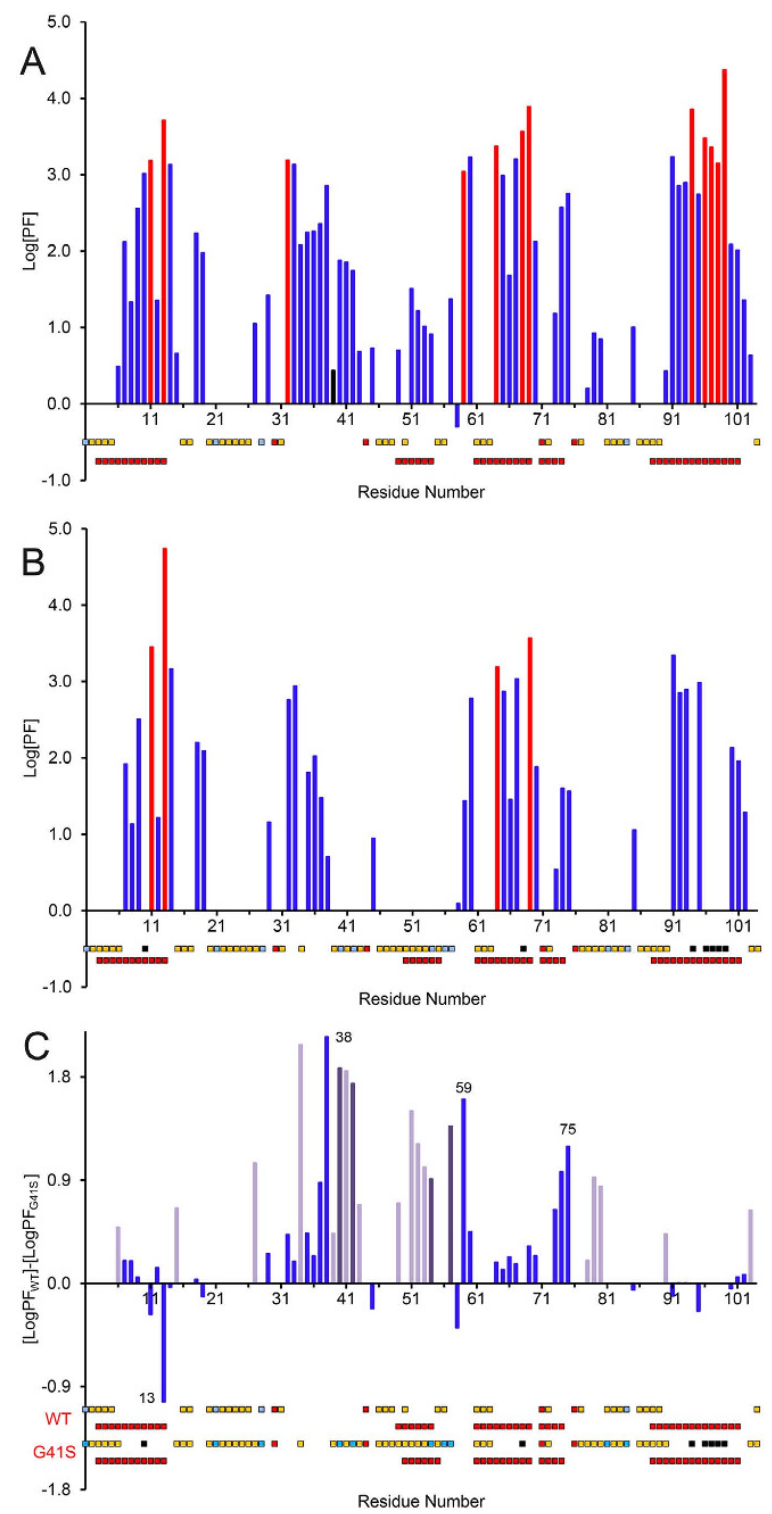

Figure 4. Backbone amide proton $H / D$ exchange analysis of $H$-ferricyt and its G41S variant. PFs for WT (A) and the G41S variant (B) plotted against the residue number. For WT, 12 residues underwent fast-exchange $(\log \mathrm{PF} \leq 1), 16$ medium $(1<\log \mathrm{PF} \leq 2), 23$ slow $(2<\log \mathrm{PF})$ and 12 extremely slow-exchange (residual proton occupancy $>80 \%$ after $\sim 68 \mathrm{~h}$, corresponding to exchange rates $<0.003 \mathrm{~h}^{-1}$, shown in red). For G41S, 4 residue underwent fast-exchange, 14 medium, 15 slow and 4 extremely slow-exchange (residual proton occupancy $>80 \%$, after $\sim 72 \mathrm{~h}$, corresponding to exchange rates $<0.003 \mathrm{~h}^{-1}$, shown in red). Secondary structure ( $\alpha$-helix) is indicated by the bottom red squares, while red squares above represent Pro residues. Unassigned and very fast-exchanging residues are indicated in light blue and yellow squares, respectively. Residues for which PFs could not be calculated due to the slowness of the exchange in the G41S variant are indicated with black squares (7 residues). (C) PF difference plot of [ $\left[\log \mathrm{P}_{\mathrm{WT}}\right]$-[ $\left[\log \mathrm{P}_{\mathrm{G} 41 \mathrm{~S}}\right]$ shown in blue. Residues that are fully exchanged in G41S have the PFs observed for WT displayed (light purple). Residues that are unassigned only for the G41S variant have the PFs observed for WT displayed (dark purple).

cyt sequences and the $\mathrm{H}$-bonds between their side chains and propionate- 7 are expected to be conserved in $90 \%$ of the same sequences ${ }^{38}$. However, there are more potential $\mathrm{H}$-bonding groups within $\mathrm{H}$-bonding distance of the heme propionates than can be simultaneously accommodated in $\mathrm{H}$-bonds ${ }^{22}$. The apparent differences in $\mathrm{H}$-bond networks in different structures suggests that some of the weaker $\mathrm{H}$-bonds are continually being broken and remade, a view that ties in with the demonstration from $\mathrm{H} / \mathrm{D}$ exchange that $40-57 \Omega$-loop has considerable dynamics $^{16,17}$. The rapid H/D exchange for the amide resonances of residues Thr40, Gln42, Gly54, Gly56 and Ile57 in the G41S variant is further evidence that this region of the variant has increased dynamics since most of these residues have significant $\mathrm{H} / \mathrm{D}$ exchange protection in the WT protein. 

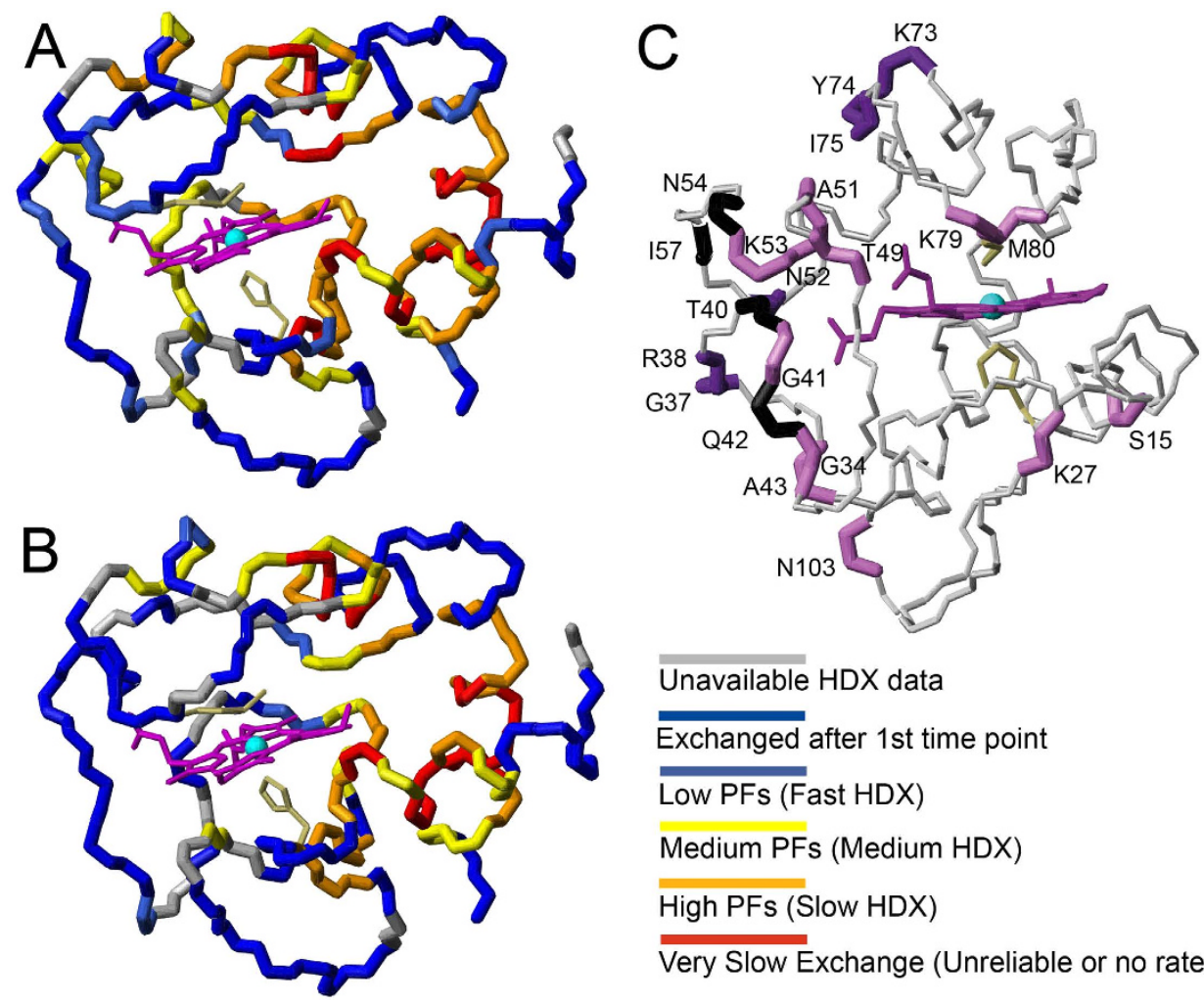

Unavailable HDX data
Exchanged after 1st time point
Low PFs (Fast HDX)
Medium PFs (Medium HDX)

High PFs (Slow HDX)

Very Slow Exchange (Unreliable or no rates)

Figure 5. PF mapping for $\mathbf{H}$-ferricyt. PFs are colour coded as indicated on (A) the WT and (B) the G41S $\mathrm{X}$-ray structures ${ }^{20,21}$. The WT cartoon representation in Fig. $1 \mathrm{~A}$ can be used to aid orientation. (C) H/D exchange difference profile mapped onto the X-ray structure of the G41S variant. Residues highlighted in violet have $\mathrm{PF}>0.5$ in WT but are fully exchanged in G41S, residues highlighted in dark violet have $\left[\Delta\left(\log \mathrm{PF}_{\mathrm{WT}^{-}}\right.\right.$ $\left.\log \mathrm{PF}_{\mathrm{G} 41 \mathrm{~S}}\right)>0.5$ ] and residues highlighted in black have $\mathrm{PF}>0.5$ in WT but are unassigned for G41S.

The G41S variant has enhanced conformational exchange in all three $\Omega$-loops. Backbone amide groups of ${ }^{15} \mathrm{~N}$-labelled proteins have been extensively used to identify regions of proteins that undergo a range of dynamics on both a ps-ns and $\mu \mathrm{s}-\mathrm{ms}$ timescale ${ }^{24}$. Out of the 96 assigned backbone resonances for the WT protein, full relaxation data were obtained for 93 residues (Fig. S4). The average relaxation rates $\left(\mathrm{R}_{1}\left(1 / \mathrm{T}_{1}\right)\right.$ and $\left.\mathrm{R}_{2}\left(1 / \mathrm{T}_{2}\right)\right)$, for the WT protein were $\mathrm{R}_{1}=0.99 \pm 0.01 \mathrm{~s}^{-1}$ and $\mathrm{R}_{2}=14.23 \pm 0.22 \mathrm{~s}^{-1}$ with an average NOE $=0.84 \pm 0.01$. Full relaxation data were obtained for all 92 assigned backbone resonances of the G41S variant (Fig. S4). The average values obtained were: $\mathrm{R}_{1}=1.07 \pm 0.03 \mathrm{~s}^{-1}, \mathrm{R}_{2}=14.48 \pm 0.38 \mathrm{~s}^{-1}$ and NOE $=0.84 \pm 0.01$. Heteronuclear $\left\{{ }^{1} \mathrm{H}\right\}{ }^{-1} \mathrm{~N}$ NOE values are indicators of motions in the ps timescale with values close to 1 suggesting rigidity and lower values indicating increased local flexibility. Both WT and the G41S variant are rigid in this respect with only the C-terminal residue E104 having a NOE value below 0.65 (Fig. 6A,B). The $\mathrm{R}_{1}$ rates are sensitive to motion on the ns to ps timescales, and are relatively constant throughout both the WT and G41S sequence. However, a handful of $\mathrm{R}_{2}$ rates for the WT are significantly increased above the average, notably those for residues Val20, Gly23, Gly56 and Asn 103, probably reflecting contributions to relaxation from slower conformational exchange contributions, as observed with other proteins (Fig. S4). Significantly more residues for the G41S variant have $\mathrm{R}_{2}$ rates deviating from the average (Fig. S4). Plots of $\mathrm{R}_{2} / \mathrm{R}_{1}$ ratios, which are a useful indicator for identifying residues experiencing chemical exchange, show that the G41S variant has significantly more residues displaying slower conformational exchange behaviour than the WT protein (Fig. 6A,C). In particular, residues in the 19-35 $\Omega$-loop (Val20, Gly23, His33, Gly34), the neck unit (Gly37, Arg38, Lys39 and Ile58), the 40-57 $\Omega$-loop (Ser41, Ser47, Ala51, Asn52, Lys53, Lys55), the 71-85 $\Omega$-loop (Gly77) and A101 and N103 at the end of the C-terminal $\alpha$-helix.

Further analysis of the relaxation data was performed with the Model-free formalism ${ }^{40}$ (see SI and Table S3), which provides three main dynamic parameters: a) the generalized order parameter, $\mathrm{S}^{2}$, b) the effective correlation time, $\tau_{e}$ and c) the chemical exchange contribution rate, $R_{e x}$. The average $S^{2}$ values, which specify the degree of spatial restriction of the N-H bond, for WT and the G41S variant are comparable at $0.924 \pm 0.046$ and $0.934 \pm 0.050$, respectively (Fig. 7). This is consistent with both proteins being relatively rigid, presumably due to the $\alpha$-helical secondary structure and the compact core held together by hydrophobic interactions associated with the heme. For WT H-ferricyt, large $\mathrm{R}_{\mathrm{ex}}$ terms (between 12 and $16 \mathrm{~s}^{-1}$ ) were observed for Val20 and Gly23 (average of $\left.14.52 \pm 1.01 \mathrm{~s}^{-1}\right)$ and smaller $\mathrm{R}_{\mathrm{ex}}$ terms $\left(<5 \mathrm{~s}^{-1}\right)$ for residues Ile9, His 18, Gly24, Lys25, Lys39, Gly41, Gly45, Ile57, Trp59, Gly60, Ile95, Tyr97 and Ala101 (average of $1.56 \pm 0.76 \mathrm{~s}^{-1}$ ) (Fig. 7A). For the G41S variant, large $\mathrm{R}_{\mathrm{ex}}$ terms (between 8 and $16 \mathrm{~s}^{-1}$ ) were observed for Val20, Gly23, His33, Ser41, Lys53, Lys55, Ile58 and Gly77 (average of $13.40 \pm 3.97 \mathrm{~s}^{-1}$ ) and smaller $\mathrm{R}_{\mathrm{ex}}$ terms $\left(<5 \mathrm{~s}^{-1}\right)$ for His18, His26, Gly37, Lys39, Gly45 and Thr49 (average of $2.58 \pm 1.29 \mathrm{~s}^{-1}$ ) (Fig. 7B). Overall, 13 residues have $\mathrm{R}_{\mathrm{ex}}$ terms of small magnitude and 2 residues large 

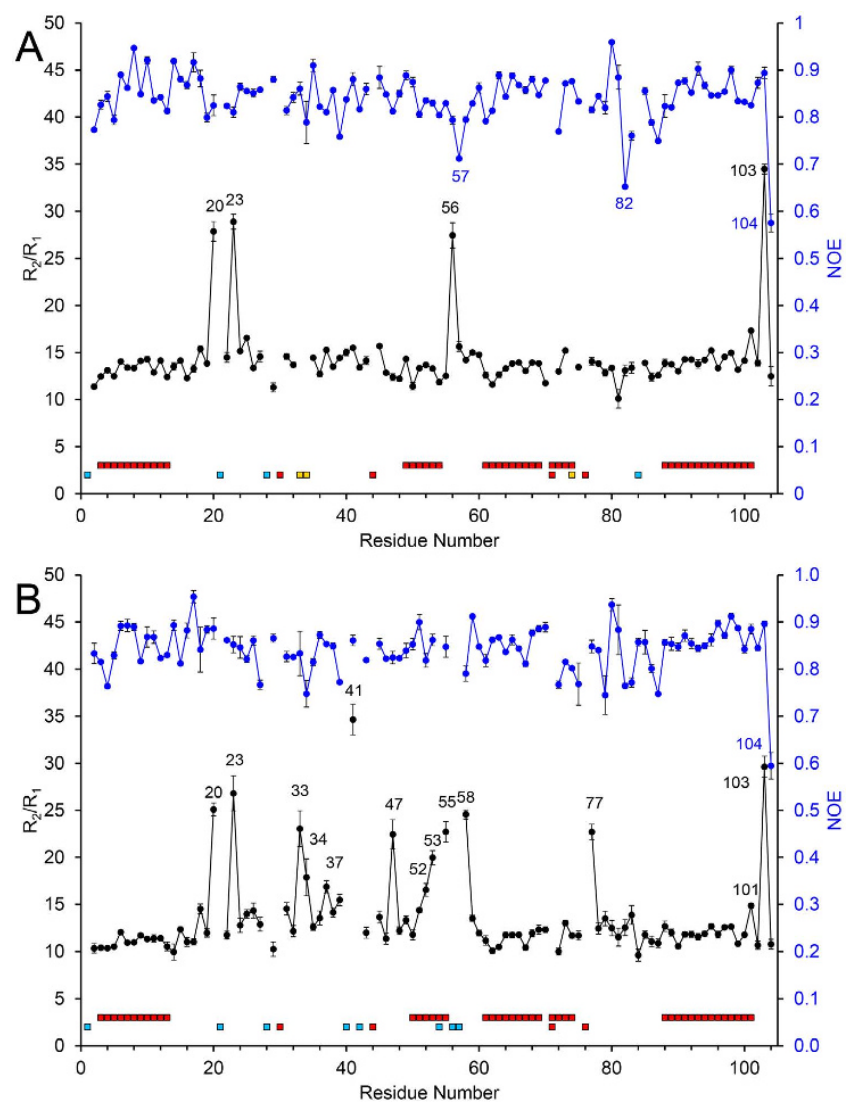

Figure 6. ${ }^{15} \mathrm{~N}$ Relaxation parameters for $\mathrm{H}$-ferricyt. $\mathrm{R}_{2} / \mathrm{R}_{1}$ (in black) and $\left\{{ }^{1} \mathrm{H}\right\}-{ }^{15} \mathrm{~N}$ NOE (in blue) for WT (A) and G41S (B). $\alpha$-helices are indicated by the top red squares while below Pro residues and unassigned residues are indicated with red and light blue squares, respectively. Residues excluded from the analysis due to peak overlap or weak intensity are indicated with yellow squares.

magnitude for WT, while for the G41S variant 6 residues have $\mathrm{R}_{\mathrm{ex}}$ terms of small magnitude and 8 residues large magnitude, thus indicating enhanced conformational exchange on the ms to $\mu$ s timescale in the G41S variant. The less informative number of residues with a correlation time $\left(\tau_{\mathrm{e}}\right)$ indicating internal motion was 23 and 20 for the WT and G41S variant, respectively (Fig. S5), with many of these assigned to model 5, which reflects both fast and slow motions (Table S3) and is difficult to accurately characterise with relaxation data. The significant finding is the number of residues exhibiting relatively large $\mathrm{R}_{\mathrm{ex}}$ terms.

The distribution of residues requiring an $\mathrm{R}_{\mathrm{ex}}$ term to describe their relaxation data for both WT (15 residues) and G41S (14 residues) H-ferricyt have been mapped onto the respective structures in Fig. 8. A striking overall resemblance to the H/D exchange difference map shown in Fig. $5 \mathrm{C}$ is observed. Furthermore it highlights that under native conditions the regions experiencing conformational exchange for both the WT and G41S proteins are essentially identical, with the switching of a glycine to a serine at position 41 enhancing the dynamics in 3 out of the $5 \mathrm{H}$-ferricyt foldons, and in particular the lowest energy foldon (40-57 $\Omega$-loop). Thus the G41S variant nicely illustrates a previous prediction that foldon substructure may determine additional properties ${ }^{16}$, such as apoptotic activity.

Conformational dynamics in the 40-57 $\Omega$-loop modulate the pentacoordinate form and apoptotic interactions. The NMR data and $\mathrm{N}_{3}{ }^{-}$binding kinetics strongly support a linkage in the dynamics of the region surrounding heme propionate-7, i.e. the $40-57 \Omega$-loop and lability of the Met 80 heme ligand. A recent $\mathrm{X}$-ray structure of the K72A variant of yeast cyt provides further compelling evidence of a direct conformational link between Met 80 and the heme propionate- 7 region by being a conformer in which the Met 80 ligand is dissociated from the heme and the Arg38 side chain has moved in a similar manner to that observed in the G41S structure (Fig. 1B) ${ }^{20,33}$. These structural changes coincide with the creation of a $\mathrm{H}_{2} \mathrm{O}$ channel leading from the vacant Met80 position to beyond heme propionate- 7 and ending at the Arg38 side chain, resembling more the conformation of the G41S H-ferricyt than the WT protein in this region. This channel, with Arg38 proposed to act as a seal, has been suggested to enable facile entry of $\mathrm{H}_{2} \mathrm{O}_{2}$ into the heme environment and/or provide a $\mathrm{H}^{+}$ exit route ${ }^{33}$. $\beta$-sheet formation in the 40 s region of the $40-57 \Omega$-loop has been reported for yeast ferricyt at low $\mathrm{pH}$ as a result of breaking a H-bond between His26-Pro44. This was suggested to trigger rearrangement in the Met80 loop since $\beta$-sheet formation was accompanied by dissociation of Met 80 from the heme iron ${ }^{41,42}$. This event also causes disruption of the H-bonding network associated with heme propionate-7 resulting in a change of the heme position relative to the polypeptide and increased mobility for aromatic residues such as Trp5941,42. A further 


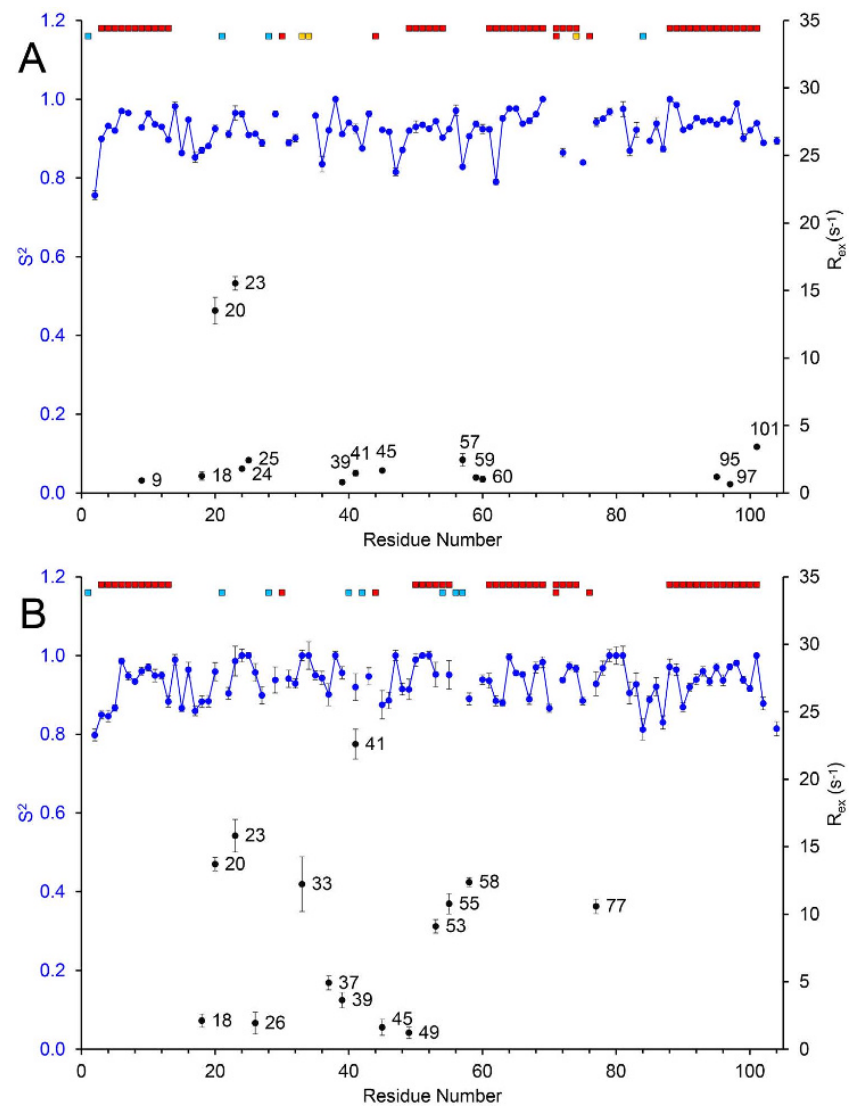

Figure 7. Model-free parameters for $\mathbf{H}$-ferricyt. $S^{2}$ order parameters (in blue) and $\mathrm{R}_{\mathrm{ex}}$ contributions (in black) for WT (A) and G41S (B). $\alpha$-helices are indicated by the top red squares while below Pro residues and unassigned residues are indicated with red and light blue squares, respectively. Residues excluded from the analysis due to peak overlap or weak intensity are indicated with yellow squares.

recent crystal structure of the T78C/K79G yeast cyt variant reveals a non-native form whereby Lys73 replaces the Met80 ligand and a conserved stretch of the Met80 loop $\left({ }^{76} \mathrm{PGTK}^{79}\right)$ refolds into a $\beta$-hairpin structure, with Gly77 being a critical element of this hairpin and central to the flexibility of this peptide fragment ${ }^{43}$. Furthermore, a positional change in helix-2 (residues 51-54) occurs to accommodate the refolded Met80 loop which has the effect of increasing the volume of the heme pocket and causing peroxidase activity to increase in this T78C/ K79G variant ${ }^{43}$. Facets of these structural changes in this non-native conformer are therefore comparable with the regions displaying increased dynamics in the G41S variant. This suggests that, in solution, alternative conformations are kinetically and thermodynamically accessible in the G41S variant, whereby the heightened dynamics in 40-57 $\Omega$-loop foldon is coupled to an increased population of a peroxidase pentacoodinate state, resulting in the elevated peroxidase activity akin to the WT protein when bound to $\mathrm{CL}^{18,19}$. In addition to the X-ray structures of the variants mentioned above, an important finding from H/D exchange studies is that the dynamics of the 40-57 $\Omega$-loop controls the rate of the alkaline isomerisation of ferricyt, which leads to Met80 being replaced by a lysine as an axial ligand ${ }^{44}$. Though the alkaline isomerisation is not a factor in our $\mathrm{N}_{3}{ }^{-}$binding experiments, and thus not a factor in generation of the pentacoordinate, it is a further demonstration that the dynamics of the 40-57 $\Omega$-loop and Met80 lability are linked and can govern function ${ }^{16}$.

Finally, the $40-57 \Omega$-loop is the least conserved in cyt evolution. This has been highlighted recently through the discovery of a species-dependent interaction between cyt and Apaf- 1 in cytosolic extracts, which is proposed to arise due to sequence variation between mitochondrial species in the $40-57 \Omega$-loop ${ }^{45}$. Further to this discovery, residues flanking Pro44 have for some time been associated with a conformational change in cells undergoing apoptosis ${ }^{46}$ and recent modelling studies suggest $\mathrm{H}$-cyt interacts with Apaf-1 through contact via the $40-57 \Omega$-loop ${ }^{45,47}$. Thus based on the present work we propose that the dynamics of $40-57 \Omega$-loop may be species-specific and tuned to regulate interaction with the cognate Apaf-1.

\section{Experimental}

NMR sample preparation. ${ }^{15} \mathrm{~N}$-labelled samples of WT H-ferricyt and the G41S variant ranging in concentration from 0.8 to $1 \mathrm{mM}$ were used for all experiments and prepared as described previously ${ }^{48}$.

NMR Spectroscopy. All NMR experiments were performed at $288 \mathrm{~K}$ (unless specified otherwise) and acquired on a Bruker $800 \mathrm{MHz}$ spectrometer equipped with either a $5 \mathrm{~mm} \mathrm{HCN}$ inverse triple resonance z-axis gradient probe or a $5 \mathrm{~mm}$ broad band inverse $\mathrm{z}$-axis gradient probe, and a Bruker $500 \mathrm{MHz}$ spectrometer 
A

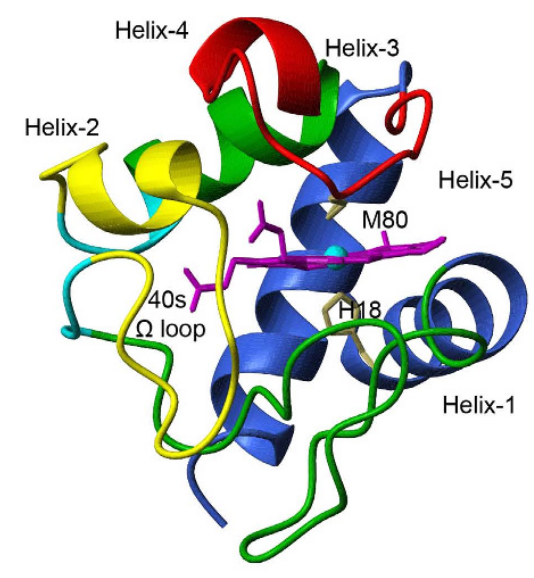

B

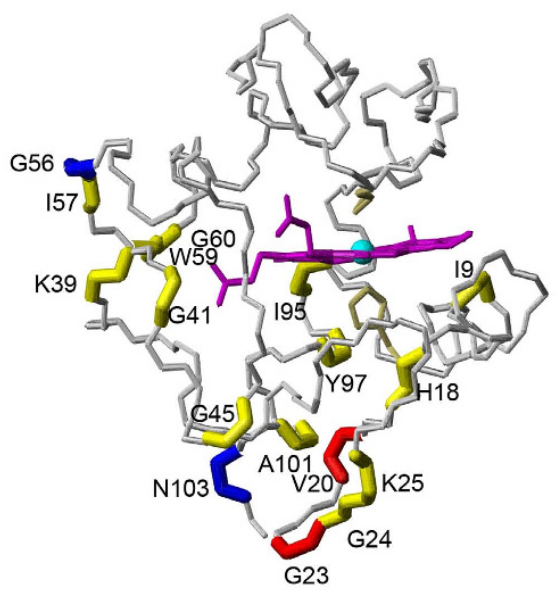

C

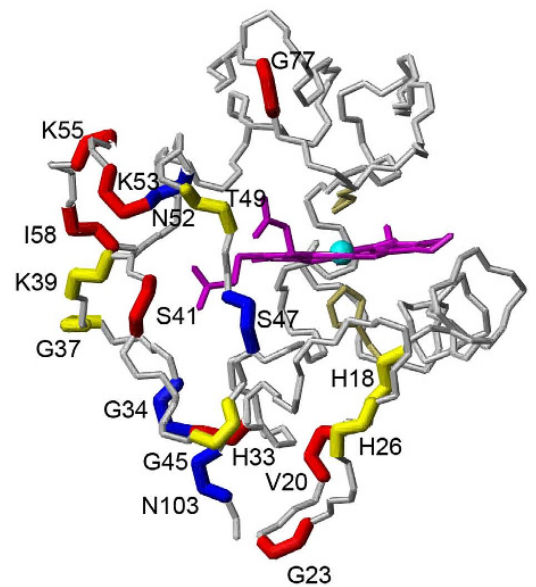

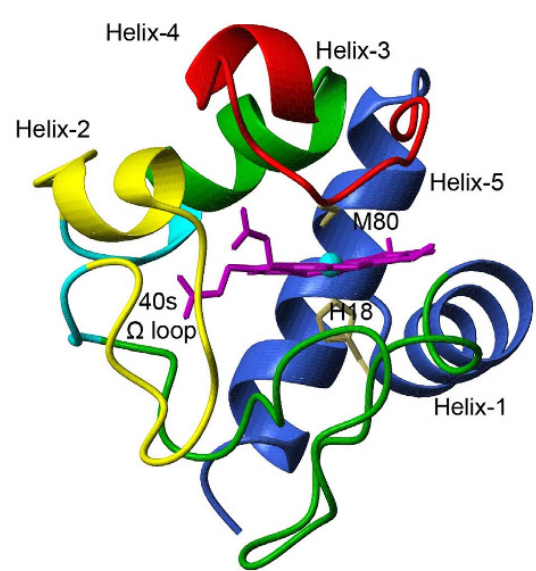
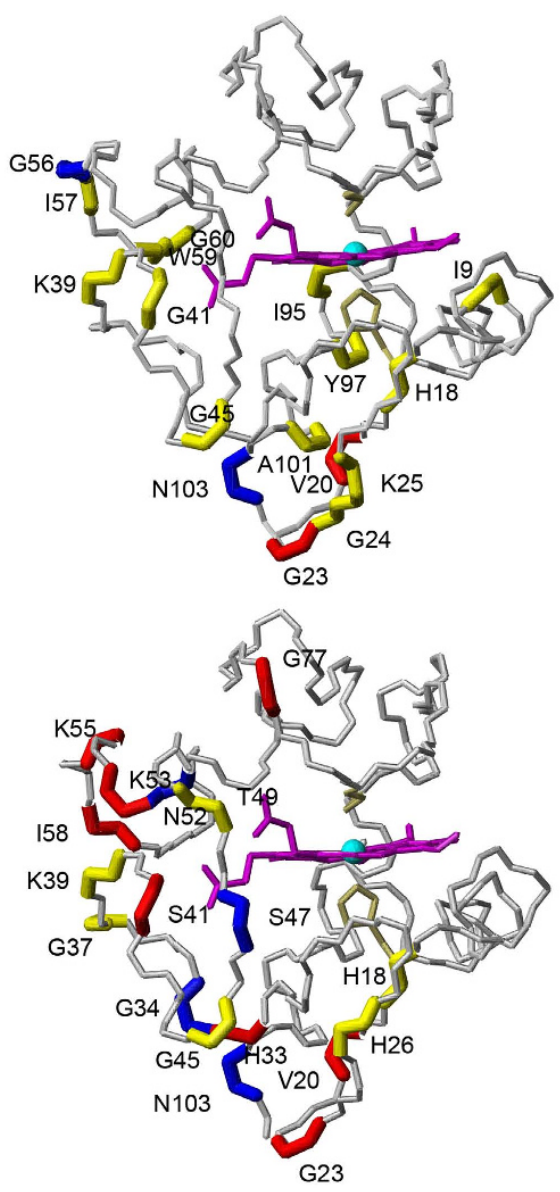

Figure 8. Dynamic profile mapping of H-ferricyt. Stereo pairs representation of (A) WT with the five foldon units coloured as described in the legend to Fig. 1A. NMR dynamics plotted on the X-ray structures of WT (B) and G41S (C). Residues highlighted in yellow and red have low $\left(<5 \mathrm{~s}^{-1}\right)$ and high $\left(>5 \mathrm{~s}^{-1}\right) \mathrm{R}_{\mathrm{ex}}$ terms, respectively, with those in blue having $R_{2} / R_{1}$ deviating from the average but without an associated $R_{e x}$ term. In all depictions heme and axial ligands are shown in stick representation.

equipped with a $5 \mathrm{~mm} \mathrm{HCN}$ inverse triple resonance z-axis gradient probe. Data were processed with either Topspin (Bruker Biospin) or the NMRPipe and NMRDraw package ${ }^{49}$. Data analysis was performed with the programs CCPNmr Analysis ${ }^{50}$ and Sparky ${ }^{51}$. The amide chemical shifts used in this work for the WT and G41S variant have been previously determined (BMRB accession numbers 25418 and 25422, respectively) ${ }^{48}$.

H/D exchange. Freeze-dried ${ }^{15} \mathrm{~N}$-labelled and desalted samples of both WT and G41S H-ferricyt at $1 \mathrm{mM}$ concentration were re-dissolved in $20 \mathrm{mM}$ sodium phosphate $\mathrm{pH} 6.5,50 \mathrm{mM} \mathrm{NaCl}$ pre-made in $99.9 \%{ }^{2} \mathrm{H}_{2} \mathrm{O}$ (Sigma). All H/D exchange NMR data were acquired at $500 \mathrm{MHz}$ as matrices of $1024 \times 64$ complex data points with 4 scans and spectral widths of $15\left({ }^{1} \mathrm{H}\right)$ and $42\left({ }^{15} \mathrm{~N}\right) \mathrm{ppm}(39.8 \mathrm{ppm}$ for G41S) centred at 4.7 and $118.6 \mathrm{ppm}$, 
respectively. The acquisition of consecutive ${ }^{1} \mathrm{H}^{15} \mathrm{~N}$-HSQC spectra started approximately 5 min after sample resuspension. Acquisition proceeded for WT with consecutive HSQC spectra of approximately 5 min acquisition time for a period of $68 \mathrm{~h}$, totalling 792 experiments. The first 49 experiments (to ensure maximum coverage of the fast-exchanging residues) were followed by 74 experiments at $50 \mathrm{~min}$ intervals, totalling 123 experiments, which were used for the extrapolation of the H/D exchange rates. For the G41S variant, acquisition of ${ }^{1} \mathrm{H}-{ }^{15} \mathrm{~N}-\mathrm{HSQC}$ spectra of identical acquisition parameters was executed at specific timed intervals, i.e. 49 consecutive experiments followed by 79 experiments at 50 min intervals. CCPNmr Analysis was used to fit peak volumes as a function of time to a two-parameter single exponential decay function ${ }^{50}$. Rates of exchange were extracted from this fitting and were used for the calculation of $\mathrm{PFs}^{35,52}$. Intrinsic exchange rate constants required for the calculation of PFs were obtained as described previously ${ }^{35} . \Delta \mathrm{G}_{\mathrm{ex}}$ (Table S1) values were obtained from $\Delta \mathrm{G}_{\mathrm{ex}}=-\mathrm{RT} \ln (\mathrm{PF})$ where $\mathrm{R}$ is the gas constant and $\mathrm{T}$ is the temperature in Kelvin ${ }^{52}$.

${ }^{15} \mathrm{~N}$ relaxation data. ${ }^{15} \mathrm{~N} \mathrm{~T}_{1}, \mathrm{~T}_{2}$, and $\left\{{ }^{1} \mathrm{H}\right\}-{ }^{15} \mathrm{~N}$ NOE data were collected at $800 \mathrm{MHz}$. Spectra were acquired as matrices of $1024 \times 256$ complex data points with 8 scans for $\mathrm{T}_{1}$ and $\mathrm{T}_{2}$ data and 64 scans for $\left\{{ }^{1} \mathrm{H}\right\}-{ }^{15} \mathrm{~N}$ NOE data. Spectral widths of $15\left({ }^{1} \mathrm{H}\right)$ and $39.3\left({ }^{15} \mathrm{~N}\right)$ ppm centred at 4.7 and $118.6 \mathrm{ppm}$ respectively, were used. $\mathrm{T}_{1}$ experiments were acquired with relaxation delays of 10, 50, 80, 200, 500, 750, 2000 and 3500 ms with duplicates at 10,200 and $500 \mathrm{~ms}$. $\mathrm{T}_{2}$ experiments were acquired with relaxation delays of $16.96,33.92,50.88,67.84,101.76$, 135.68, 203.52 and $254.40 \mathrm{~ms}$ with duplicates at 16.96, 50.88 and $101.76 \mathrm{~ms}$. Recycle delays for both experiments were $5 \mathrm{~s}$. The repeated relaxation delays were used for the determination of peak height uncertainties ${ }^{53}$. Identical delays were used for both WT and G41S with the exception of the first $\mathrm{T}_{1}$ delay for G41S, which was $20 \mathrm{~ms}$ due to pulse sequence limitations. Both $\mathrm{T}_{1}$ and $\mathrm{T}_{2}$ series of experiments were acquired with interleaved delay values to minimize sample heating. $\mathrm{T}_{1}$ and $\mathrm{T}_{2}$ data for G41S were acquired as a pseudo-3D experiment, and processed as individual $2 \mathrm{D}$ planes using NMRPipe ${ }^{49} . \mathrm{T}_{1}$ and $\mathrm{T}_{2}$ values were extracted through the fitting of peak heights as a function of the relaxation delay to a two-parameter single exponential decay function using the follow intensity changes function of CCPN Analysis. These were subsequently converted to $R_{1}$ and $R_{2}$ rate constants (R, where $R_{1}=1 / T_{1}$ and $R_{2}=1 / T_{2}$ ). The fit errors for $T_{1}$ and $T_{2}$ were also converted into $R_{1}$ and $R_{2}$ errors through the equality of the $T_{1} / T_{2}$ and $R_{2} / R_{1}$ errors. Heteronuclear $\left\{{ }^{1} H\right\}-{ }^{15} \mathrm{~N}$ NOEs were measured with a proton saturation period of $5 \mathrm{~s}$. Interleaved saturated and unsaturated experiments were acquired in triplicate in order to determine the experimental errors as the standard deviation of the average NOE value. NOE values were calculated as the ratio of the peak heights with and without proton saturation.

Model-free analysis. Model-free analysis was performed using the Model-free 4 program ${ }^{54}$ complemented with the FAST-Modelfree program ${ }^{55}$ to facilitate and speed-up data analysis and interpretation. The first step consisted of the estimation of the rotational correlation time using the program r2r1_tm (http://www.palmer. hs.columbia.edu/) from the relaxation data. The program quadric_diffusion (http://www.palmer.hs.columbia. $\mathrm{edu} /$ ) was used to calculate the rotational diffusion parameters from the output local correlation times. This program uses a $3 \mathrm{D}$ structure translationally centred on the centre of mass and rotated to the inertia tensor principal axes. These manipulations were achieved with the program pdbinertia (http://www.palmer.hs.columbia. $\mathrm{edu} /$ ). Initial pdb manipulations preceding the use of pdbinertia were performed with molecular graphics program MOLMOL ${ }^{56}$. Quadric_diffusion also produces a pdb structure rotated to axially symmetric principal axes, subsequently used as an input for FAST-Model-free. The paramagnetic contribution from the low-spin $(S=1 / 2)$ heme to the nitrogen relaxation rates is considered negligible because of the low gyromagnetic ratio of the ${ }^{15} \mathrm{~N}$ nucleus ${ }^{57}$. The Tjandra-Bax conditions ${ }^{58}$ were applied in order to exclude residues with a large-amplitude fast internal motions and residues subject to conformational exchange from the rotational correlation time calculations. The former applies if the $\left\{{ }^{1} \mathrm{H}\right\}-{ }^{15} \mathrm{~N} N O E<0.65$ and the later applies if $\left[\left(\mathrm{T}_{2, \mathrm{av}}-\mathrm{T}_{2, \mathrm{n}}\right) / \mathrm{T}_{2, \mathrm{av}}-\left(\mathrm{T}_{1, \mathrm{av}}-\mathrm{T}_{1, \mathrm{n}}\right) /\right.$ $\mathrm{T}_{2 \mathrm{av}} \mathrm{J}>1.5 \mathrm{x}$ standard deviation, where $\mathrm{T}_{1, \mathrm{av}}$ and $\mathrm{T}_{2, \mathrm{av}}$ the average values, and $\mathrm{T}_{1, \mathrm{n}}$ and $\mathrm{T}_{2, \mathrm{n}}$ are the relaxation times for each individual residue, $\mathrm{n}$. These conditions identify the 'rigid' residues with relaxation parameter values corresponding to the global rotational dynamics.

The five models considered for the interpretation of dynamic parameters using the Model-free approach ${ }^{40,59,60}$ were: model 1 (with only the generalized order parameter $S^{2}$ ), model 2 (with $S^{2}$ and the correlation time for internal motion $\tau_{e}$ ), model 3 ( $S^{2}$ and the exchange contribution to the relaxation rate, $R_{e x}$, or model 1 with $R_{e x}$ ), model $4\left(S^{2}, \tau_{e}\right.$ and $R_{e x}$ or model 2 with $R_{e x}$ ) and finally the extended model 5 (with the generalized order parameter for faster and slower timescales, $S^{2}=S^{2}{ }_{f}^{2}{ }_{s}$ and $\tau_{e}$ ) which accounts for a fast and a slow internal motion. The models with $\mathrm{R}_{\mathrm{ex}}$ terms ( 3 and 4 ) reflect on conformational exchange processes on the ms to $\mu$ s timescale. The models with effective correlation time reflect on internal motions on the ps to ns timescale. Model-free analysis was performed with ${ }^{15} \mathrm{~N}$ gyromagnetic ratio of $-2.71, \mathrm{~N}-\mathrm{H}$ bond distance of $1.02 \AA$ and ${ }^{15} \mathrm{~N}$ chemical shift anisotropy of -160 . FAST-Model-free calculations were performed with confidence limit for $\chi^{2}$-testing of 0.95 , confidence limit for F-testing of 0.80, 50 iterations, sum squared error cut-off of 200, 500-1000 Monte-Carlo simulations and optimization of the diffusion tensor.

Stopped-flow kinetics. Kinetic experiments were carried out using an Applied Photophysics (Leatherhead, UK) SX20 stopped-flow spectrophotometer equipped with both photomultiplier and diode array detection systems and thermostatted at $25^{\circ} \mathrm{C}$. Stocks of sodium azide $2 \mathrm{M}$ were prepared in $50 \mathrm{mM}$ sodium phosphate and $50 \mathrm{mM}$ MES pH 7 and diluted to the desired $\mathrm{N}^{3-}$ concentration with the same buffer but containing $2 \mathrm{M} \mathrm{NaCl}$ to maintain the ionic strength. Time courses were taken at $420 \mathrm{~nm}$ with known $\mathrm{N}^{3-}$ ] (between $0.08-2 \mathrm{M}$ before mixing) and $10 \mu \mathrm{M}$ protein (before mixing) with the transient fitted to a single exponential function yielding both pseudo first-order rate constants and amplitudes. All errors reported are standard errors. 


\section{References}

1. Li, P. et al. Cytochrome $c$ and dATP-dependent formation of Apaf-1/caspase-9 complex initiates. Cell 91, 479-489 (1997).

2. Kagan, V. E. et al. Cytochrome $c$ acts as a cardiolipin oxygenase required for release of proapoptotic factors. Nat Chem Biol $\mathbf{1}$, 223-232 (2005).

3. Huttemann, M. et al. The multiple functions of cytochrome $c$ and their regulation in life and death decisions of the mammalian cell: From respiration to apoptosis. Mitochondrion 11,369-381, doi: 10.1016/j.mito.2011.01.010 (2011).

4. Gonzalez-Arzola, K. et al. Structural basis for inhibition of the histone chaperone activity of SET/TAF-Ibeta by cytochrome $c$. Proc Natl Acad Sci USA 112, 9908-9913, doi: 10.1073/pnas.1508040112 (2015).

5. Diederix, R. E., Ubbink, M. \& Canters, G. W. Peroxidase activity as a tool for studying the folding of $c$-type cytochromes. Biochemistry 41, 13067-13077 (2002).

6. Sutin, N. \& Yandell, J. K. Mechanisms of reactions of cytochrome $c$ - Rate and equilibrium constants for ligand binding to horse heart ferricytochrome c. J Biol Chem. 247, 6932-6936 (1972).

7. Vladimirov, Y. A., Proskurnina, E. V. \& Alekseev, A. V. Molecular mechanisms of apoptosis. structure of cytochrome $c$-cardiolipin complex. Biochemistry-Moscow 78, 1086-1097, doi: 10.1134/s0006297913100027 (2013).

8. Rosell, F. I., Ferrer, J. C. \& Mauk, A. G. Proton-linked protein conformational switching: Definition of the alkaline conformational transition of yeast iso-1-ferricytochrome c. J Am Chem Soc 120, 11234-11245, doi: 10.1021/ja971756+ (1998).

9. Wilson, M. T. Cytochrome c: A multidisciplinary approach. (University Science Books, 1996).

10. Morison, I. M. et al. A mutation of human cytochrome $c$ enhances the intrinsic apoptotic pathway but causes only thrombocytopenia. Nat Genet 40, 387-389 (2008).

11. De Rocco, D. et al. Mutations of cytochrome $c$ identified in patients with thrombocytopenia THC4 affect both apoptosis and cellular bioenergetics. Biochim Biophys Acta 1842, 269-274, doi: 10.1016/j.bbadis.2013.12.002 (2014).

12. Hunte, C., Koepke, J., Lange, C., Rossmanith, T. \& Michel, H. Structure at $2.3 \AA$ resolution of the cytochrome bc(1) complex from the yeast Saccharomyces cerevisiae co-crystallized with an antibody Fv fragment. Structure 8, 669-684, doi: 10.1016/S09692126(00)00152-0 (2000).

13. Lange, C. \& Hunte, C. Crystal structure of the yeast cytochrome bc(1) complex with its bound substrate cytochrome $c$. Proc Natl Acad Sci USA 99, 2800-2805, doi: 10.1073/pnas.052704699 (2002).

14. Sakamoto, K. et al. NMR basis for interprotein electron transfer gating between cytochrome $c$ and cytochrome $c$ oxidase. Proc Natl Acad Sci USA 108, 12271-12276, doi: 10.1073/pnas.1108320108 (2011).

15. Volkov, A. N., Nicholls, P. \& Worrall, J. A. R. The complex of cytochrome $c$ and cytochrome $c$ peroxidase: The end of the road? Biochim Biophys Acta 1807, 1482-1503, doi: 10.1016/j.bbabio.2011.07.010 (2011).

16. Krishna, M. M., Lin, Y., Rumbley, J. N. \& Englander, S. W. Cooperative omega loops in cytochrome $c$ : role in folding and function. J Mol Biol 331, 29-36 (2003).

17. Krishna, M. M., Maity, H., Rumbley, J. N., Lin, Y. \& Englander, S. W. Order of steps in the cytochrome $c$ folding pathway: evidence for a sequential stabilization mechanism. J Mol Biol 359, 1410-1419, doi: 10.1016/j.jmb.2006.04.035 (2006).

18. Rajagopal, B. S., Silkstone, G. G., Nicholls, P., Wilson, M. T. \& Worrall, J. A. R. An investigation into a cardiolipin acyl chain insertion site in cytochrome c. Biochim Biophys Acta 1817, 780-791, doi: 10.1016/j.bbabio.2012.02.010 (2012).

19. Josephs, T. M., Morison, I. M., Day, C. L., Wilbanks, S. M. \& Ledgerwood, E. C. Enhancing the peroxidase activity of cytochrome $c$ by mutation of residue 41: implications for the peroxidase mechanism and cytochrome $c$ release. Biochem J 458, 259-265, doi: 10.1042/bj20131386 (2014).

20. Liptak, M. D., Fagerlund, R. D., Ledgerwood, E. C., Wilbanks, S. M. \& Bren, K. L. The proapoptotic G41S mutation to human cytochrome $c$ alters the heme electronic structure and increases the electron self-exchange rate. J Am Chem Soc 133, 1153-1155, doi: $10.1021 /$ ja106328k (2011).

21. Rajagopal, B. S. et al. The hydrogen-peroxide-induced radical behaviour in human cytochrome $c$-phospholipid complexes: implications for the enhanced pro-apoptotic activity of the G41S mutant. Biochem J 456, 441-452, doi: 10.1042/bj20130758 (2013).

22. Moore, G. R. \& Pettigrew, G. W. Cytochrome $c$ : Evolutionary,structural and physicochemical aspects. Springer-Verlag, London (1990).

23. Josephs, T. M. et al. Conformational change and human cytochrome $c$ function: mutation of residue 41 modulates caspase activation and destabilizes Met-80 coordination. J Biol Inorg Chem, doi: 10.1007/s00775-012-0973-1 (2013).

24. Akke, M. NMR methods for characterizing microsecond to millisecond dynamics in recognition and catalysis. Curr Opin Struct Biol 12, 642-647 (2002)

25. Henzler-Wildman, K. \& Kern, D. Dynamic personalities of proteins. Nature 450, 964-972 (2007).

26. Fetrow, J. S. \& Baxter, S. M. Assignment of ${ }^{15} \mathrm{~N}$ chemical shifts and ${ }^{15} \mathrm{~N}$ relaxation measurements for oxidized and reduced iso-1cytochrome $c$. Biochemistry 38, 4480-4492 (1999).

27. Barker, P. D. et al. A further clue to understanding the mobility of mitochondrial yeast cytochrome $c$ : a ${ }^{15} \mathrm{~N}$ T1rho investigation of the oxidized and reduced species. Eur J Biochem 268, 4468-4476 (2001).

28. Sakamoto, K., Kamiya, M., Uchida, T., Kawano, K. \& Ishimori, K. Redox-controlled backbone dynamics of human cytochrome $c$ revealed by ${ }^{15} \mathrm{~N}$ NMR relaxation measurements. Biochem Biophys Res Commun 398, 231-236, doi: 10.1016/j.bbrc.2010.06.065 (2010).

29. Rafferty, S. P., Smith, M. \& Mauk, A. G. Azide binding and active site dynamics of position-82 variants of ferricytochrome $c$. Inorg Chim Acta 242, 171-177, doi: 10.1016/0020-1693(95)04863-4 (1996).

30. Winkler, J. R. Cytochrome c folding dynamics. Curr Opin Chem Biol 8, 169-174 (2004).

31. Borutaite, V. \& Brown, G. C. Mitochondrial regulation of caspase activation by cytochrome oxidase and tetramethylphenylenediamine via cytosolic cytochrome $c$ redox state. J Biol Chem 282, 31124-31130 (2007).

32. Diederix, R. E., Ubbink, M. \& Canters, G. W. The peroxidase activity of cytochrome $c_{550}$ from Paracoccus versutus. Eur J Biochem 268, 4207-4216 (2001).

33. McClelland, L. J., Mou, T. C., Jeakins-Cooley, M. E., Sprang, S. R. \& Bowler, B. E. Structure of a mitochondrial cytochrome $c$ conformer competent for peroxidase activity. Proc Natl Acad Sci USA 111, 6648-6653, doi: 10.1073/pnas.1323828111 (2014).

34. Englander, S. W. \& Kallenbach, N. R. Hydrogen-exchange and structural dynamics of proteins and nucleic-acids. Q Rev Biophys 16, 521-655 (1983)

35. Bai, Y., Milne, J. S., Mayne, L. \& Englander, S. W. Primary structure effects on peptide group hydrogen exchange. Proteins 17, 75-86 (1993).

36. Milne, J. S., Mayne, L., Roder, H., Wand, A. J. \& Englander, S. W. Determinants of protein hydrogen exchange studied in equine cytochrome c. Protein Sci 7, 739-745 (1998).

37. Baxter, S. M. \& Fetrow, J. S. Hydrogen exchange behavior of $\left[\mathrm{U}-{ }^{15} \mathrm{~N}\right]$-labeled oxidized and reduced iso-1-cytochrome $c$. Biochemistry 38, 4493-4503 (1999).

38. Banci, L., Bertini, I., Rosato, A. \& Varani, G. Mitochondrial cytochromes $c$ : a comparative analysis. J Biol Inorg Chem 4, 824-837 (1999).

39. Maity, H., Maity, M., Krishna, M. M., Mayne, L. \& Englander, S. W. Protein folding: the stepwise assembly of foldon units. Proc Natl Acad Sci USA 102, 4741-4746, doi: 10.1073/pnas.0501043102 (2005).

40. Lipari, G. \& Szabo, A. Model-Free Approach to the interpretation of nuclear magnetic-resonance relaxation in macromolecules.1. Theory and range of validity. J Am Chem Soc 104, 4546-4559, doi: 10.1021/ja00381a009 (1982). 
41. Balakrishnan, G. et al. A conformational switch to beta-sheet structure in cytochrome $c$ leads to heme exposure. Implications for cardiolipin peroxidation and apoptosis. J Am Chem Soc 129, 504-505, doi: 10.1021/ja0678727 (2007).

42. Balakrishnan, G., Hu, Y. \& Spiro, T. G. His26 protonation in cytochrome $c$ triggers microsecond beta-sheet formation and heme exposure: implications for apoptosis. J Am Chem Soc 134, 19061-19069, doi: 10.1021/ja307100a (2012).

43. Amacher, J. F. et al. A compact structure of cytochrome $c$ trapped in a lysine-ligated state: Loop refolding and functional implications of a conformational switch. J Am Chem Soc 137, 8435-8449, doi: 10.1021/jacs.5b01493 (2015).

44. Maity, H., Rumbley, J. N. \& Englander, S. W. Functional role of a protein foldon-an Omega-loop foldon controls the alkaline transition in ferricytochrome c. Proteins 63, 349-355, doi: 10.1002/prot.20757 (2006).

45. Josephs, T. M., Hibbs, M. E., Ong, L., Morison, I. M. \& Ledgerwood, E. C. Interspecies variation in the functional consequences of mutation of cytochrome c. PloS one 10, e0130292, doi: 10.1371/journal.pone.0130292 (2015).

46. Jemmerson, R. et al. A conformational change in cytochrome $c$ of apoptotic and necrotic cells is detected by monoclonal antibody binding and mimicked by association of the native antigen with synthetic phospholipid vesicles. Biochemistry 38, 3599-3609, doi: 10.1021/bi9809268 (1999).

47. Shalaeva, D. N., Dibrova, D. V., Galperin, M. Y. \& Mulkidjanian, A. Y. Modeling of interaction between cytochrome $c$ and the WD domains of Apaf-1: bifurcated salt bridges underlying apoptosome assembly. Biol. Direct 10, 29, doi: 10.1186/s13062-015-0059-4 (2015).

48. Karsisiotis, A. I. et al. Backbone resonance assignments of ferric human cytochrome $c$ and the pro-apoptotic G41S mutant in the ferric and ferrous states. Biomol NMR Assign 9, 415-419, doi: 10.1007/s12104-015-9621-3 (2015).

49. Delaglio, F. et al. NMRPipe: a multidimensional spectral processing system based on UNIX pipes. J Biomol NMR 6, 277-293 (1995).

50. Vranken, W. F. et al. The CCPN data model for NMR spectroscopy: development of a software pipeline. Proteins 59, 687-696, doi: 10.1002/prot.20449 (2005).

51. Goddard, T. D. \& Kneller, D. G. SPARKY 3. University of California, San Francisco (2008)

52. Hoshino, M., Katou, H., Yamaguchi, K.-I. \& Goto, Y. Dimethylsulfoxide-quenched hydrogen/deuterium exchange method to study amyloid fibril structure. Biochim Biophys Acta 1768, 1886-1899, doi: 10.1016/j.bbamem.2007.03.001 (2007).

53. Palmer, A. G., Rance, M. \& Wright, P. E. Intramolecular motions of a Zinc finger DNA-binding domain from Xfin characterized by proton-detected natural abundance C-12 heteronuclear NMR-Spectroscopy. J Am Chem Soc 113, 4371-4380, doi: 10.1021/ ja00012a001 (1991)

54. Mandel, A. M., Akke, M. \& Palmer, A. G., 3rd. Backbone dynamics of Escherichia coli ribonuclease HI: correlations with structure and function in an active enzyme. J Mol Biol 246, 144-163 (1995).

55. Cole, R. \& Loria, J. P. FAST-Modelfree: a program for rapid automated analysis of solution NMR spin-relaxation data. J Biomol NMR 26, 203-213 (2003).

56. Koradi, R., Billeter, M. \& Wuthrich, K. MOLMOL: A program for display and analysis of macromolecular structures. J Mol Graph 14, 51-\&, doi: 10.1016/0263-7855(96)00009-4 (1996).

57. Ubbink, M., Worrall, J. A. R., Canters, G. W., Groenen, E. J. \& Huber, M. Paramagnetic resonance of biological metal centers. Annu Rev Biophys Biomol Struct 31, 393-422 (2002).

58. Tjandra, N., Feller, S. E., Pastor, R. W. \& Bax, A. Rotational diffusion anisotropy of human ubiquitin from ${ }^{15} \mathrm{~N}$ NMR relaxation. J Am Chem Soc 117, 12562-12566, doi: 10.1021/ja00155a020 (1995).

59. Lipari, G. \& Szabo, A. Model-Free approach to the interpretation of nuclear magnetic-resonance relaxation in macromolecules.2. Analysis of experimental results. J Am Chem Soc 104, 4559-4570, doi: 10.1021/ja00381a010 (1982).

60. Clore, G. M. et al. Deviations from the simple 2-parameter Model-Free approach to the interpretation of ${ }^{15} \mathrm{~N}$ nuclear magneticrelaxation of proteins. J Am Chem Soc 112, 4989-4991, doi: 10.1021/ja00168a070 (1990).

\section{Acknowledgements}

This work was supported by a Leverhulme Trust project grant (RPG-2013-164) to JARW and a Leverhulme Trust emeritus fellowship (EM-2014-088) to GRM.

\section{Author Contributions}

A.I.K. and O.M.D. prepared all protein samples. A.I.K. recorded and analysed all NMR data. O.M.D. and M.T.W. carried out the stopped-flow kinetics and analysed the data. C.M. and T.M.A.B. assisted with NMR data collection. G.R.M. and J.A.R.W. designed and supervised the project and wrote the manuscript, with contributions from all authors.

\section{Additional Information}

Supplementary information accompanies this paper at http://www.nature.com/srep

Competing financial interests: The authors declare no competing financial interests.

How to cite this article: Karsisiotis, A. I. et al. Increased dynamics in the 40-57 $\Omega$-loop of the G41S variant of human cytochrome $c$ promote its pro-apoptotic conformation. Sci. Rep. 6, 30447; doi: 10.1038/srep30447 (2016).

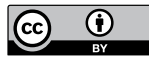

This work is licensed under a Creative Commons Attribution 4.0 International License. The images or other third party material in this article are included in the article's Creative Commons license, unless indicated otherwise in the credit line; if the material is not included under the Creative Commons license, users will need to obtain permission from the license holder to reproduce the material. To view a copy of this license, visit http://creativecommons.org/licenses/by/4.0/

(c) The Author(s) 2016 